\title{
Evaluation of anti-fibrotic activity of ethanolic extract of Nelumbo nucifera gaertn. Seed against doxorubicin and unilateral ureter obstruction-induced renal fibrosis
}

\author{
R Rushendran', Dr. V Jayasankar Reddy², T Bharath Kumar', T Mamatha, J Roja', T Roopavani ${ }^{3}$ \\ ${ }^{1}$ Department of Pharmacology, Krishna teja pharmacy college, Tirupati, Andhra Pradesh, India \\ 2 Professor, HOD, Department of Pharmacology, Krishna teja pharmacy college, Tirupati, Andhra Pradesh, India \\ ${ }^{3}$ Department of Pharmacognosy, Sree Vidyanikethan Pharmacy College, Tirupati, Andhra Pradesh, India
}

Rushendran R, Jayasankar Reddy V, Bharath Kumar T, Mamatha T, Roja J, Roopavani T. Evaluation of anti-fibrotic activity of ethanolic extract of Nelumbo nucifera gaertn. Seed against doxorubicin and unilateral ureter obstruction-induced renal fibrosis. J Pre-Clin Clin Res. 2017; 11(1): 66-75. doi: 10.26444/jpccr/75319

\section{Abstract}

Objective. The study were undertaken to evaluate anti-fibrotic activity of ethanolic extract of Nelumbo nucifera seed (NNSEE) against doxorubicin and Unilateral Ureter Obstruction-induced renal fibrosis.

Materials and method. Animals were divided into five groups with six animals in each group, i.e. Normal, Positive control, Standard (Pirfenidone 200mg/kg), Test-I (NNSEE 200mg/kg), and Test-II (NNSEE 400mg/kg). Renal fibrosis was developed by doxorubicin and UUO-induced methods. After induction of renal fibrosis, profibrotic responses in biochemical parameters were observed, e.g. BUN, serum creatinine levels were elevated, while total protein and GFR levels decreased. Antioxidant (SOD and CAT) levels are also attenuates and hyalinized glomeruli cells, damage to tubular cells were noted in histopathology, which are all responsible for the development of renal fibrosis.

Results. The result showed that the anti-fibrotic activity of NNSEE at a dose of 200 and $400 \mathrm{mg} / \mathrm{kg}$ b.wt was comparable with the standard treatment $200 \mathrm{mg} / \mathrm{kg}$ b.wt of pirfenidone (anti-fibrotic drug). These data supplemented with histopathological studies of rat kidney sections. NNSEE had reversed all the manifestation of renal fibrosis.

Conclusion. Results of the study indicate that the NNSEE has potent anti-fibrotic activity, as well as antioxidant property, in dose dependent manner that may be due to the presence of major phytochemical constituents such as alkaloids, polyphenols.

\section{Key words}

renal fibrosis, anti-fibrosis, Nelumbo nucifera, pirfenidone, kidney

\section{INTRODUCTION}

For human beings, health is most important from birth to end of life, but from the past to the present man has been suffering from diseases. Although global modernization has bought about a revolutionary change in the field of medicine for diagnosis, treatment and understanding disease, but the present day sophisticated life style, food habits, nature of work, etc. are leading to a number of life-threatening diseases. Nowadays, renal fibrosis has become one of the most problematic diseases has been suffering throughout the world.

Renal fibrosis occurs when the normal tissue architecture is slowly taken place by an extracellular matrix (ECM). Fibrosis of both glomerular and tubulointerstitial compartments correlates with diminishing renal function in all progressive forms of diabetic and non-diabetic glomerular disease which leads to end-stage renal disease. Renal fibrosis is characterized by the excessive deposition of connective tissue and organ inflammation. Literature reviews by the authors of the presented study suggest that the main reason for renal fibrosis has revealed a close relationship between the expression of TGF- $\beta$ and cytokine. TGF- $\beta$ is predominantly present in human umbilical vein endothelial cells. The main role of TGF- $\beta$ is increasing the ECM protein synthesis, fibronectin,

Address for correspondence: R Rushendran, Department of Pharmacology in Krishna teja pharmacy college

E-mail:rushiyuva@gmail.com

Received: 9 January 2017; accepted: 19 May 2017 and also promote the accumulation of ECM. It was revealed recently that the TGF- $\beta$ expression is upregulated in proliferative and fibrotic glomerular lesions in various humans and experimental nephropathies, glomerulonephritis and diabetic nephropathy [1]. This condition impacts on millions of people worldwide and can lead to the deterioration of the kidney, which leads to death. In fact, about $40 \%$ of all deaths due to kidney failure are linked to fibrosis. Kidney fibrosis typically occurs in every type of CKD, a medical condition characterized by the gradual loss of kidney function over time [2]. Allopathic medications are safe and efficacious, but prolonged usage may cause disabling side-effects. In order to remedy this kind of condition, using the herbal medicine is very effective, rather than the allopathic drugs.

The World Health Organization (WHO) reports that $80 \%$ of the world population presently use herbal medicine for initial health care. During the past few decades, the affinity for herbal drugs has grown by the utilization of traditional medicinal plants to heal some critical diseases. It is proving to be better medicine with respect to synthetic drugs that assure numerous side-effects by long-term therapy. In recent years, focus on plant research has increased worldwide. A large body of evidence has been collected that shows the immense potential of medicinal plants used in various traditional systems [3].

Although there are many plants like Apiumgraveolens, Boerhaviadiffusa, Phyllanthusniruri, Datura metal which have medicinal properties, Hibiscus sabdariffaetc has a nephroprotective property which has been proved scientifically. 
Traditionally using the Nelumbo nucifera plant, which also has the nephroprotective property, especially the seeds having anti-fibrotic property has not been scientifically proved todate. Therefore, based on the phytochemical constituents present in Nelumbo nucifera seed, an attempt was made in the presented study to evaluate the anti-fibrotic activity against doxorubicin and UUO-induced renal fibrosis, which show a more potent therapeutic efficacy than the present use of anti-fibrotic drugs.

\section{MATERIALS AND METHOD}

Plant materials and authentication. Dried seeds of Nelumbo nucifera were collected from an ayurveda store in Tirupati, Chittoor district, Andhra Pradesh, India, in January 2016. The plant Nelumbo nucifera was identified and authentified by Dr. K. Madhava Chetty, Assistant Professor in the Department of Botany at Sri Venkateswara University, Tirupati, Chittoor district, India. A voucher specimen was submitted in the Krishna Teja Pharmacy College, Tirupati, Andhra Pradesh, India. The plant material was air dried indoors at room temperature.

Plant extraction. The collected dried seeds of Nelumbo nucifera were cleaned and powdered in a grinding mill. The seed powder was shaken in 44-mesh sieve for the coarse fined powder. The fined powder was stored in airtight container at room temperature until preparation of extract. In the extraction process, two solvents were used - petroleum ether and ethanol. The fine-powdered drug was subjected to extraction of petroleum ether solvent in 1:4 ratios (w/v), by using Soxhlet apparatus for 12 hours to remove fat material, and the marc was reused for extraction with $70 \%$ ethanol at $40^{\circ} \mathrm{C}$. The extraction was carried out until the extract becames colourless. Extract was concentrated, dried and packed in an air-tight container. The obtained semi-solid extract of Nelumbo nucifera seed was used for animal studies and phytochemical screening, stored at a cool temperature of $2-4^{\circ} \mathrm{C}$, throughout the duration of the study [4].

Phytochemical estimation. The preliminary phytochemical estimation was carried out by Pet. ether and NNSEE for qualitative identification of phytochemical constituents, such as alkaloids, carbohydrates, gums, proteins and amino acids, glycosides, steroids and flavonoids by using standard procedures.

a) Detection of alkaloids. Extracts were dissolved individually in dil. HCL and filtered.

Mayer's test: Filtrate was treated with Mayer's reagent (Potassium mercuric Iodide) which forms a yellowcoloured precipitate indicating the presence of alkaloids. Wagner's test. Filtrate was treated with Wagner's reagent (Iodide in Potassium Iodide) which forms a brown or reddish-coloured precipitate indicating the presence of alkaloids.

b) Detection of flavonoids - Alkaline reagent test. Extracts were treated with a few drops of sodium hydroxide solution which turns a yellow colour indicating the presence of flavonoids, and by adding dilute acid becomes colourless.
Lead acetate test. Extracts were treated with a few drops of lead acetate solution and formed a yellow colour precipitate indicating the presence of flavonoids.

c) Detection of carbohydrates. Extracts were dissolved individually in a few $\mathrm{ml}$ of distilled water and filtered. Molisch's test. Filtrates were treated with two drops of alcoholic $\alpha$-naphthol solution which formed a violet ring at the junction, indicating the presence of carbohydrates.

Benedict's test. Filtrates were treated with Benedict's reagent and heated gently. An orange red precipitate indicated the presence of carbohydrates.

d)Detection of proteins and amino acids.

Xanthoproteic acid. Extracts were treated with a few drops of conc. nitric acid and formed a yellow colour indicating the presence of proteins.

Ninhydrin test. $0.25 \% \mathrm{w} / \mathrm{v}$ ninhydrin reagent was added to the extract and boiled for a few minutes, forming a blue colour indicating the presence of amino acid.

\section{e) Detection of saponins.}

Forth test: Extracts were diluted with distilled water $(20 \mathrm{ml})$ and shaken well in a graduated cylinder for $15 \mathrm{~min}$. Formation of a $1 \mathrm{~cm}$ layer of foam indicated the presence of saponins.

f) Detection of tannins.

Gelatin test. $1 \%$ gelatin solution containing sodium chloride was added to the extract. A white precipitate formed indicating the presence of tannins.

\section{g) Detection of phenols.}

Ferric chloride test. Extracts were treated with 3-4 drops of ferric chloride solution which formed a bluishblack colour indicating the presence of phenols.

\section{h) Detection of glycosides.}

Modified Borntrager's test. Extracts were treated with ferric chloride solution and immersed in boiling water for approx. $5 \mathrm{~min}$. The mixture was cooled and extracted with an equal volumes of benzene. The benzene layer was separated and treated with ammonia solution which formed a rose-pink colour in the ammonical layer, indicating the presence of glycosides.

Legal's test. Extracts were treated with sodium nitropruside in pyridine and sodium hydroxide and changed colour from pink to red, indicating the presence of glycosides.

\section{i) Detection of Polysterols.}

Salkowski's test. Extracts were treated with chloroform and filtered; the filtrate was treated with few drops of conc. sulfuric acid, shaken well, and allowed it to stand for a few mins. The appearance of a golden yellow colour indicate the presence of polysterols.

Liebermann Buchard's test. Extracts were treated with chloroform and filtered; the filtrate was treated with few drops of acetic anhydride, boiled and cooled. Conc. sulfuric acid was added which formed a brown ring at the junction indicating the presence of polysterols (PrashantTiwariet al., 2011). 
Test animals. Healthy Wistar albino rats $(180-200 \mathrm{~g})$ were used in the study. Animals were procured from the animal house of our institute, acclimatized to laboratory conditions, i.e. housed in polypropylene cages, 12:12hours light/dark cycle, $24 \pm 2{ }^{\circ} \mathrm{C}$ and relative humidity $50 \pm 5 \%$. They were provide with standard feed pellet (Hindustan Lever Ltd., Bangalore) and drinking water ad libitum throughout the study period (OECD Guidelines, 2001). The animals were fasted for $12 \mathrm{~h}$ prior to experimentation.

All the animal studies were performed according to the rules and regulations in the guidelines of the CPCSEA (Registered No. IAEC/1521/PO/Re/S/2011/CPCSEA).

Acute toxicity studies. An acute toxicity study was carried out to demonstrate the appropriate safe dose range that could be used for subsequent experiments rather than to provide complete toxicity data on the extract. As per the OECD 423 Guidelines, Wistar albino rats (180-200g) were used for the toxicity studies. Healthy rats were randomly assigned to 4 groups with 3 rats in each group. Rats were administered with NNSEE of 5,50, 300 and 2,000mg/kg, b.wt. Signs or symptoms of acute toxicity and mortality rate were observed continuously for the first $4 \mathrm{~h}$, followed by $24 \mathrm{~h}$, and further observation prolonged for up to 14 days.

\section{METHODOLOGY}

\section{Doxorubicin inducing method}

Body system. Antineoplastic and Immunomodulating agents.

Schedule: Chemotherapy type.

Doxorubicin hydrochloride $10 \mathrm{mg} / 5 \mathrm{ml}$ injection, $5 \mathrm{ml}$ vial. Doxorubicin hydrochloride $50 \mathrm{mg} / 25 \mathrm{ml}$ injection, $25 \mathrm{ml}$ vial. Doxorubicin hydrochloride $100 \mathrm{mg} / 50 \mathrm{ml}$ injection, $50 \mathrm{ml}$ vial.

Animals are injected intravenously with a single dose of doxorubicin $4 \mathrm{mg} / \mathrm{kg}$. The rate of renal damage and development of fibrosis increases with the increase in doxorubicin dose. To examine the extent of disease, this can be stratified on the basis of the proteinuria levels. The renal damage starts after 1-2 weeks and progresses continuously for several weeks. During this period of time, renal functions drop and ECM proteins gradually accumulate in the glomeruli and tubulointerstitium.

At the end of the studied time, the animals were sacrificed and the kidneys are examined for tubular injury, inflammation and fibrosis using histopathological analysis. Biochemical parameter such as glomerular filtration rate, serum creatinine, blood urea nitrogen (BUN) and total protein levels were assess. This model was also used to evaluate with Pirfenidone (200mg/kg b.wt, p.o) and NNSEE.

Unilateral ureter obstruction method. Animals were anesthetized with general anesthesia with ketamine chloride (50mg/kg b.wt i.p). The left kidney was exposed via flank incision and the ureter ligated with silk suture (i.e 4-0) for reliable ligation. Abdominal muscle and skin were stitched separately, layer-by-layer. Renal fibrosis was visible on day 3 at the earliest, and extensive fibrosis occured after 2-3 weeks.

This animal model was from 3 days to 21 days, but 14 days is an optimum time for testing anti-fibrotic drugs. At the end of the studied time point, the animals were sacrificed and the kidneys are examined for tubular injury, inflammation and fibrosis, using histopathological analysis. Biochemical parameters like glomerular filtration rate, serum creatinine, blood urea nitrogen (BUN) and total protein levels were assess. This model has been used to evaluate with Pirfenidone (200mgkg, b.wt) and NNSEE[5].

Antioxidant studies - Catalase (CAT). Catalase activity was measured by the method of Aebi (1974). [6].

\section{Calculation:}

$$
\text { Catalase }=\log \left(\frac{\mathrm{A}}{\mathrm{B}}\right) \times 2297.3
$$

where:

A - Initial absorbance

B - Final absorbance (after 30 seconds)

Units $=\mu$ moles of $\mathrm{H}_{2} \mathrm{O}_{2}$ consumed $/ \mathrm{min} / \mathrm{mg}$

Superoxide dismutase (SOD). Superoxide dismutase (SOD) activity was determined by the method developed by Kakkar et al, 1984. [7].

\section{Calculation:}

$$
\mathrm{SOD}=\frac{0.025-\mathrm{Y}}{\mathrm{Y} \times 50} \times 100
$$

where:

$\mathrm{Y}=$ Final reading - initial reading

Histopathology. Kidney tissues were fixed in $10 \%$ formalin for at least 24 hours, embedded in paraffin and cut into $3 \mu \mathrm{m}$ thick sections in rotary microtome. The section was stained withhematoxylin and eosin, observed under a microscope to detect histopathological changes in the kidney and photographs were taken.

Statistical analysis. Results were presented as mean \pm SEM $(n=6)$. The data was statistically analysed by One-way analysis of variance (ANOVA), followed by Dunnett's multiple comparison tests ( $\mathrm{t}$ test) and ${ }^{\mathrm{a}} \mathrm{P}<0.05,{ }^{\mathrm{b}} \mathrm{P}<0.01,{ }^{\mathrm{c}} \mathrm{P}<0.001$ were considered as significant, $\mathrm{P}>0.05$ was considered as nonsignificant (ns) which was compared with positive control. All statistical analyses were performed with Graphpad Prism software application (7.01version).

\section{RESULTS}

\section{Priliminary phytochemical test}

More phytochemical constituents are present in NNSEE (alkaloids, flavonoids, tannins, saponins, carbohydrates, phenols, glycosides, proteins and amino acids) than in the Pet. ether extract. Therefore, in the subsequent studies NNSEE was used because it may show better therapeutic efficacy than the petroleum ether extract due to the presence of most of the biochemical constituents.

\section{Acute toxicity studies}

The animals were observed individually after administration of NNSEE, at first 6 hours periodically during the first 24 hours, and thereafter daily for a total 14 days. There was no 
change in the normal behavioural pattern of rats; no signs and symptoms of toxicity were observed. NNSEE were safe upto a maximum dose of $2,000 \mathrm{mg} / \mathrm{kg}$ b.wt. The biological testing was carried out at doses of 200 and $400 \mathrm{mg} / \mathrm{kg}$ b.wt of NNSEE. The acute toxicity results of NNSEE are tabulated below.

\section{Biochemical parameters}

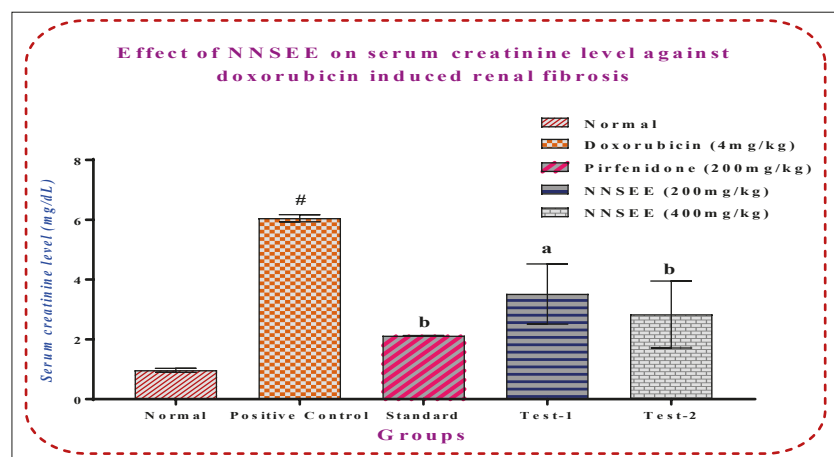

Graph 1. Estimation of serum creatinine level in doxorubicin-induced renal fibrosis. Data represent the mean \pm SEM, $(n=6)$. Statistical significance a $p<0.05,{ }^{b} p<0.01$, $p<0.001$, was compared with positive control group (One-way ANOVA followed by Dunnett's multiple comparisons test).

Graph 1 shows the serum creatinine level (mg/dL) among different groups $(n=6)$, compared with positive control (\#). Doxorubicin-induced in the rats resulted in a signifcant increase in serum creatinine level, compared with normal group. Elevated creatinine level may lead to one of the reasonable profibrotic responses. Rats were then treated with standard Pirfenidone (200mgkg, b.wt) and NNSEE (200mg/ $\mathrm{kg}$ and $400 \mathrm{mg} / \mathrm{kg}$ ), which attenuates the profibrotic responses. NNSEE and pirfenidone, which ameliorates renal dysfunction in rats against doxorubicin-induced renal fibrosis. Finally, it was observed that the test- 2 of NNSEE showed better results than the test- 1 .

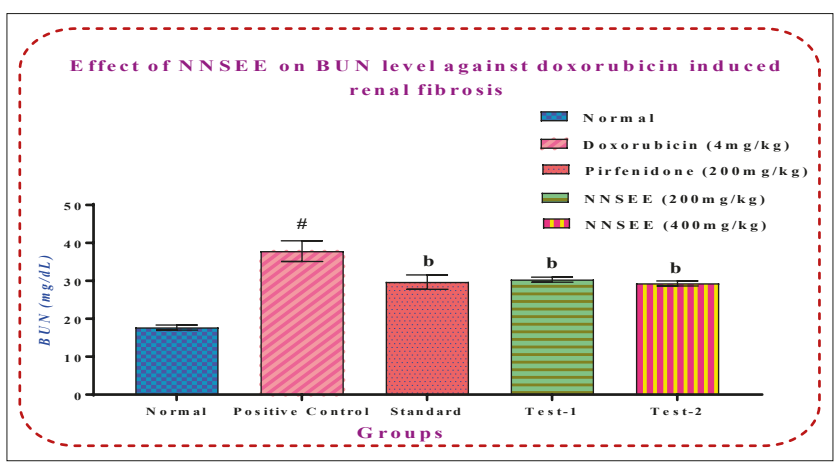

Graph 2. Estimation of BUN level in doxorubicin-induced renal fibrosis. Data represent the mean $\pm S E M,(n=6)$. Statistical significance a $p<0.05,{ }^{b} p<0.01$, ${ }^{c} p<0.001$, was compared with positive control group (One-way ANOVA followed by Dunnett's multiple comparisons test).

Graph 2 shows that the BUN level (mg/dL) among different groups $(n=6)$ compared with positive control (\#). In this estimation, when compared with the normal group, the BUN level had increased in doxorubicin-induced rats. The abnormal BUN level may result in profibrotic responses. Rats were then treated with standard (Pirfenidone $200 \mathrm{mg} / \mathrm{kg}$ )and NNSEE $(200 \mathrm{mg} / \mathrm{kg}$ and $400 \mathrm{mg} / \mathrm{kg})$, which attenuates the elevated BUN levels. NNSEE and pirfenidone ameliorates renal dysfunction in rats with doxorubicin-induced renal

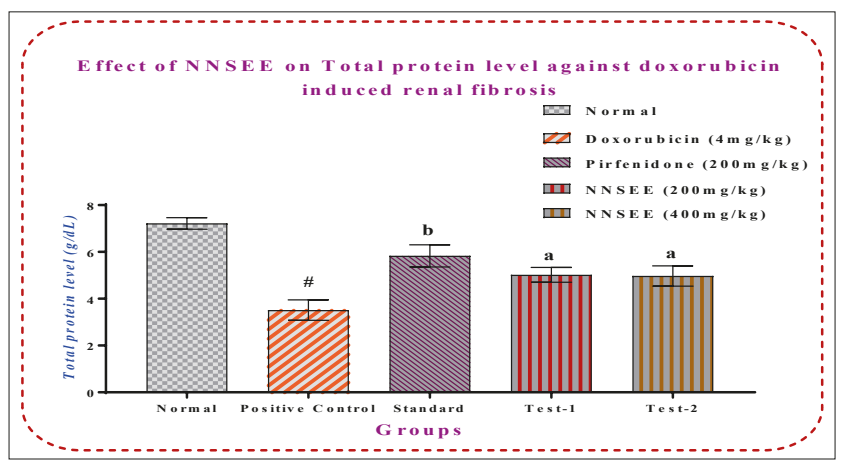

Graph 3. Estimation of total protein levels in doxorubicin-induced renal fibrosis. Data represent the mean \pm SEM, $(n=6)$. Statistical significance a $p<0.05,{ }^{b} p<0.01$, ${ }^{c} p<0.001$, was compared with positive control group (One-way ANOVA followed by Dunnett's multiple comparisons test).

fibrosis. Finally, it was observed that the test- 2 dose of NNSEE showed better results than the test-1.

Graph 3 shows the total protein level (g/dL) among different groups $(n=6)$ compared with positive control $(\#)$. Total protein levels were decreased in doxorubicin-induced rats when compared with normal group. Reduced levels of total protein content may lead to profibrotic responses. The rats were next treated with standard(Pirfenidone $200 \mathrm{mg} / \mathrm{kg}$ ) and NNSEE $(200 \mathrm{mg} / \mathrm{kg}$ and $400 \mathrm{mg} / \mathrm{kg}$ ), which ameliorates the decreased levels of total protein. NNSEE and pirfenidone ameliorate renal dysfunction in rats with doxorubicininduced renal fibrosis. Finally, it was observed that the test2 dose of NNSEE showed better results than the test-1.

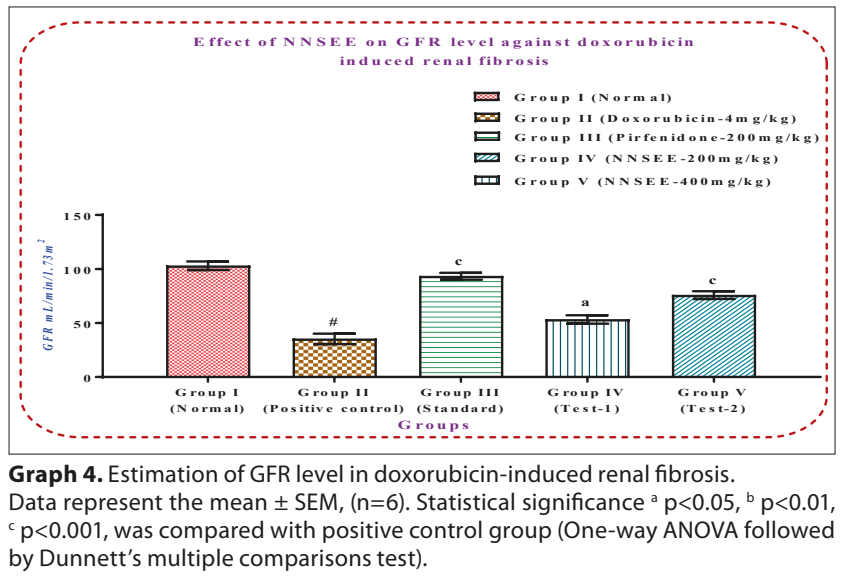

Graph 4 shows the GFR level $\left(\mathrm{mL} / \mathrm{min} / 1.73 \mathrm{~m}^{2}\right)$ among different groups $(n=6)$ compared with positive control (\#). The GFR level signifcantly decreased when treated with doxorubicin. Reduced GFR level shows renal dysfunction and may lead to profibrotic responses. Rats were then treated with standard (Pirfenidone $200 \mathrm{mg} / \mathrm{kg}$ ) andNNSEE (200mg/kg and $400 \mathrm{mg} / \mathrm{kg}$ ) which reversed the effect of doxorubicin. NNSEE and pirfenidone ameliorate renal fibrosis in doxorubicininduced rats, indicating anti-fibrotic activity. Finally, it was observed that the test-2 dose of NNSEE showed better results than the test-1.

Graph 5 shows that the serum creatinine level (mg/dL) among different groups $(n=6)$ compared with positive control (\#). Serum creatinine level was signifcantly raised due to ligation of the ureter. An elevated creatinine level may lead to profibrotic responses. The rats were then treated with standard (Pirfenidone 200mg/kg) and NNSEE $(200 \mathrm{mg} / \mathrm{kg}$ 


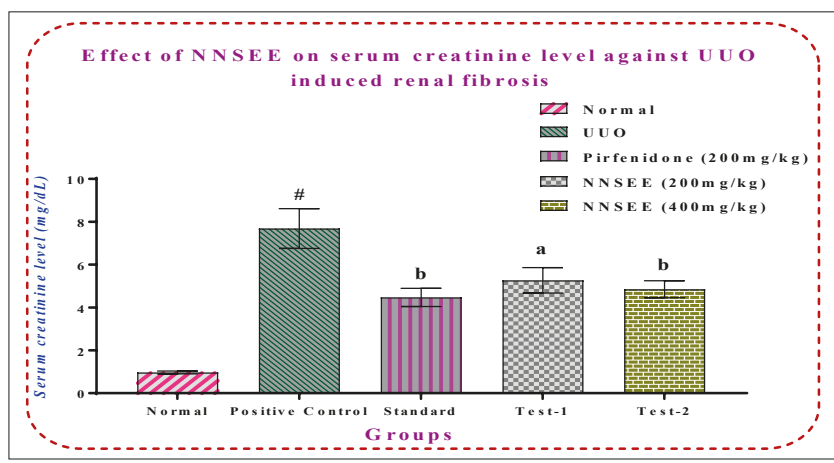

Graph 5. Estimation of serum creatinine level inUUO-induced renal fibrosis. Data represent the mean \pm SEM, $(n=6)$. Statistical significance a $p<0.05,{ }^{b} p<0.01$ c $p<0.001$, was compared with positive control group (One-way ANOVA followed by Dunnett's multiple comparisons test).

and $400 \mathrm{mg} / \mathrm{kg}$ ) which attenuates the elevated levels indicatig anti-fibrosis activity. NNSEE and pirfenidone ameliorate renal dysfunction in rats against UUO-induced renal fibrosis. Finally, it was noticed that the test- 2 dose of NNSEE showed better results than the test-1.

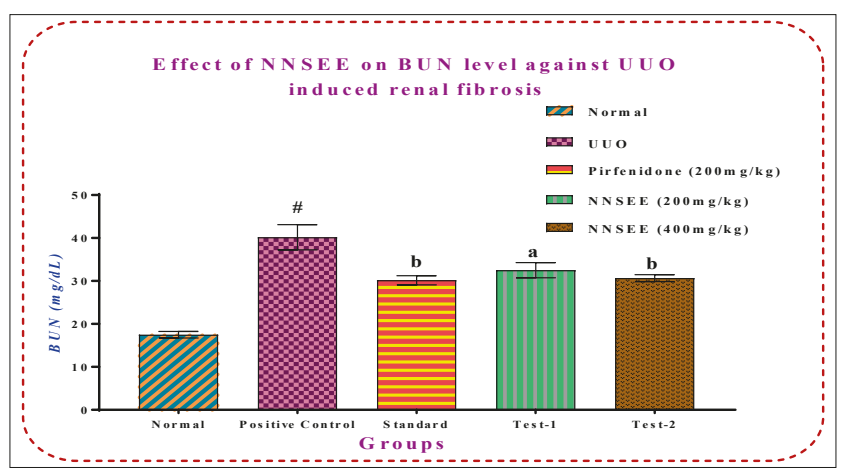

Graph 6. Estimation of BUN level in UUO-induced renal fibrosis.

Data represent the mean \pm SEM, $(n=6)$. Statistical significance ${ }^{a} p<0.05,{ }^{b} p<0.01$, $c p<0.001$, was compared with positive control group (One-way ANOVA followed by Dunnett's multiple comparisons test).

Graph 6 shows the BUN level (mg/dL) among different groups $(n=6)$ compared with positive control (\#). The BUN level was significantly raised after ligation of the right ureter. Elevated BUN level reduces renal function which leads to profibrotic responses. The rats were next treated with standard (Pirfenidone $200 \mathrm{mg} / \mathrm{kg}$ ) andNNSEE $(200 \mathrm{mg} / \mathrm{kg}$ and $400 \mathrm{mg} / \mathrm{kg}$ ) which reduces the elevated levels of BUN. NNSEE and pirfenidone ameliorates renal dysfunction in rats with UUO induced renal fibrosis. Finally, it was observed that the test-2 dose of NNSEE showed better results than the test-1.

Graph 7 shows the total protein level (g/dL) among different groups $(n=6)$ compared with positive control (\#) in UUO-induced rats when compared with the normal group. Abnormal levels of total protein content may lead to a profibrotic response. Rats were then treated with standard (Pirfenidone $200 \mathrm{mg} / \mathrm{kg}$ ) and NNSEE (200mg/kg and $400 \mathrm{mg} / \mathrm{kg}$ ) which ameliorates the decreased levels, indicating anti-fibrotic activity. NNSEE and pirfenidone ameliorates renal dysfunction in rats with UUO-induced renal fibrosis. Finally, it was noted that the test-2 dose of NNSEE showed better results than the test-1.

Graph 8 shows the GFR level $\left(\mathrm{mL} / \mathrm{min} / 1.73 \mathrm{~m}^{2}\right)$ among different groups $(n=6)$ compared with positive control (\#). When compared with the normal group, GFR level signifcantly decreased in UUO-induced rats. An abnormal

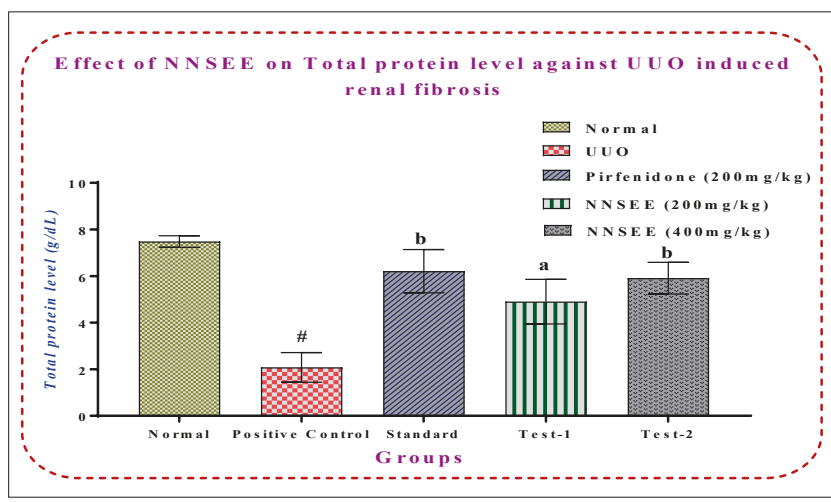

Graph 7. Estimation of Total protein level in UUO induced renal fibrosis. Data represent the mean \pm SEM, $(n=6)$. Statistical significance ${ }^{a} p<0.05,{ }^{b} p<0.01$, c $p<0.001$, was compared with positive control group (One way ANOVA followed by Dunnett's multiple comparisons test).

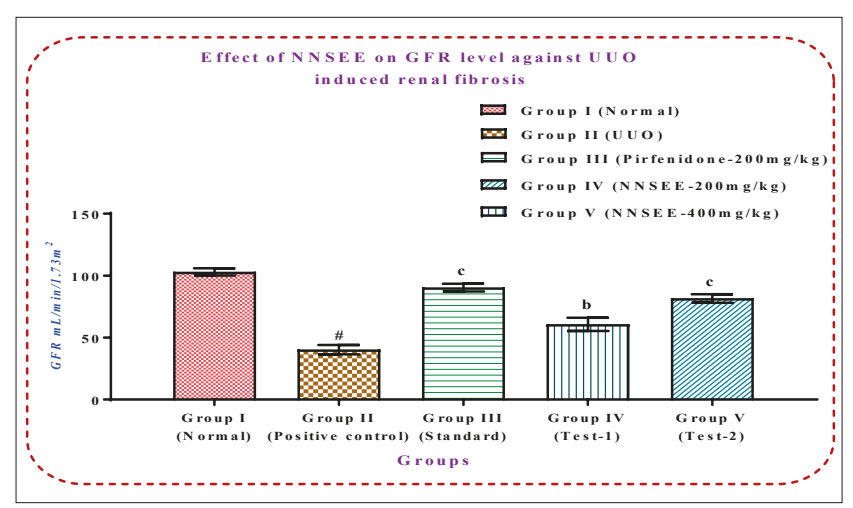

Graph 8. Estimation of GFR level in UUO induced renal fibrosis.

Data represent the mean \pm SEM, $(n=6)$. Statistical significance a $p<0.05,{ }^{b} p<0.01$, c $p<0.001$, was compared with positive control group (One-way ANOVA followed by Dunnett's multiple comparisons test).

GFR level in urine indicated renal dysfuntion which may lead to fibrosis. Rats were then treated with standard (Pirfenidone $200 \mathrm{mg} / \mathrm{kg}$ ) and NNSEE (200mg/kg and $400 \mathrm{mg} / \mathrm{kg}$ ) which ameliorates the GFR levels. NNSEE and pirfenidone(standard drug) ameliorates renal fibrosis in rats, which indicates antifibrotic activity. Finally, it was noted that the test- 2 dose of NNSEE showed better results than the test-1.

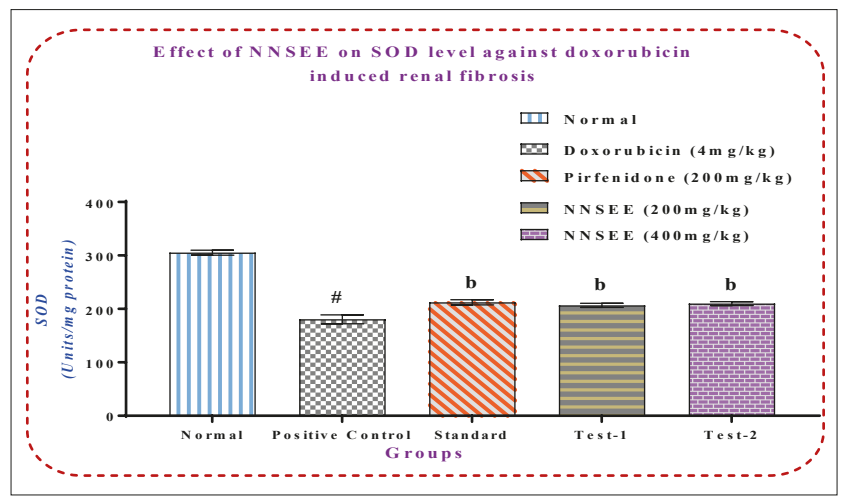

Graph 9. Estimation of SOD in doxorubicin-induced renal fibrosis.

Data represent the mean $\pm S E M,(n=6)$. Statistical significance ${ }^{a} p<0.05,{ }^{b} p<0.01, c$ $p<0.001$, was compared with positive control group (One-way ANOVA followed by Dunnett's multiple comparisons test).

Graph 9 shows the SOD (units/mg protein) level among different groups $(n=6)$ compared with positive control groups (\#). In the present study, the antioxidant acitvity 
of Superoxide dismutase (SOD) enzyme (scavenger of free radicals) in doxorubicin-induced rats was investiated. Free radicals are responsible for the profibrotic responses. In the positive control group, the SOD enzymes decreased, i.e superoxides were elevated, compared to the normal group followed by the remaining groups. Treating the rats with standard (Pirfenidone $200 \mathrm{mg} / \mathrm{kg}$ ) and NNSEE extract $(200$ and $400 \mathrm{mg} / \mathrm{kg}$ b.wt) increases the SOD enzyme which attenuates free radical activity. NNSEE and pirfenidone ameliorates renal fibrosis in doxorubicin-induced rats, indicating anti-fibrotic activity dose dependently. Finally, it was observed that test- 2 of NNSEE ( $400 \mathrm{mg} / \mathrm{kg}$ b.wt) showed better therapeutic action than the test-1 (200mg/kg b.wt).

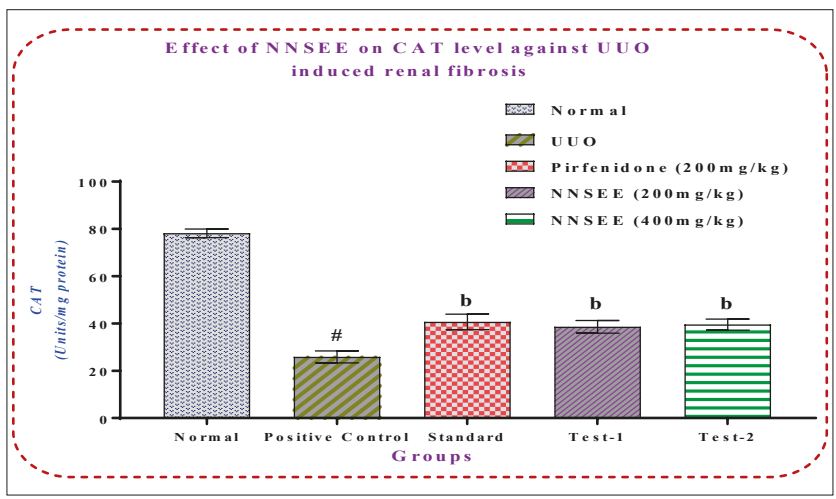

Graph 10. Estimation of CAT in UUO-induced renal fibrosis.

Data represent the mean \pm SEM, $(n=6)$. Statistical significance a $p<0.05,{ }^{b} p<0.01$, $p<0.001$, was compared with positive control group (One-way ANOVA followed by Dunnett's multiple comparisons test).

Graph 10 shows the CAT (units/mg protein) level among different groups $(n=6)$ compared with the positive control group (\#). The presented study investigated the antioxidant acitvity of catalase (CAT) enzyme (scavenger of free radicals) in UUO-induced rats. Free radicals are responsible for the profibrotic responses. In the positive control group, the catalse enzyme decreased compared to the normal group. Treating the rats with standard (Pirfenidone $200 \mathrm{mg} / \mathrm{kg}$ ) and NNSEE extract (200 and $400 \mathrm{mg} / \mathrm{kg}$ b.wt) increased the catalase enzyme which attenuates free radical activity. NNSEE and pirfenidoneameliorates renal fibrosis in rats against UUO-induced rats indicated anti-fibrotic activity dose dependently. Ultimately, noticed that test-2 of NNSEE (400mg/kg b.wt) has shown better therapeutic action than the test-1 (200mg/kg b.wt).

\section{HISTOPATHOLOGICAL STUDIES}

\section{Histopathology of UUO induced renal fibrosis}

\author{
Group I (Normal) \\ * Normal bowman's capsule. \\ * No degenerative changes. \\ * Normal cyto-architecture kidney tissue.
}

\section{Group II (UUO)}

* Degenertive changes takes place in the kidney tissue and shows necrosis.

* Vacuolization occurs.

* Hyalinized glomeruli cells are obeserved.

* Inflammatory cells are noted.
Group III (Pirfenidone 200mg/kg b.wt)

* Regenerative changes take place in the kidney tissue and shows similar to normal cyto-architecture.

Group IV (NNSEE low dose 200mg/kg b.wt)

* Regenerative changes took place.

* Hyalinized glomeruli cells were observed..

* NNSEE attenuated approximately $75-80 \%$ of the hyalinized glomeruli in kidney.

Group V(NNSEE high dose 400mg/kg b.wt)

* Regenerative changes took place in the kidney tissue and showed similar to standard cyto-architecture.

* Hyalinized glomeruli were observed although less compared to the test-1 group.

* NNSEE attenuated approximately $95 \%$ of hyalinized glomeruli in kidney.

* NNSEE showed better results than the Test-1 group.

UUO-induced kidney injury can lead to progressive renal fibrosis. In the presented study, hyalinized glomeruli cells or scar formation occuring in the kidney was assessed by Hematoxyline and eosin staining. The result of UUOinduced model is the production of massive scar formation of glomeruli cells observed in the positive control group. These fibrotic changes in UUO injured kidney was remarkably abrogated by NNSEE and standard drug, indicating antifibrotic activity. Collectively, these data suggest that the NNSEE treatment improves the long-term outcome of injured kidney by ameliorating renal fibrosis.

\section{Histopathology of doxorubicin-induced renal fibrosis}

\author{
Group I (Normal) \\ * Normal Bowman's capsule. \\ * No degenerative changes. \\ * Normal cyto-architecture kidney tissue.
}

\section{Group II (UUO) and showed necrosis. \\ * Vacuolization occurred. \\ * Tubular necrosis or damage obeserved. \\ * Inflammatory cells noted.}

* Degenertive changes took place in the kidney tissue

Group III (Pirfenidone 200mg/kg b.wt)

* Regenerative changes took place in the kidney tissue and showed similar to normal cyto-architecture.

Group IV (NNSEE low dose 200mg/kg b.wt)

* Regenerative changes took place.

* Tubular damaged cells observed.

* NSEE attenuated approximately $70-80 \%$ of tubular necrosis in kidney.

Group V(NNSEE high dose 400mg/kg b.wt)

* Regenerative changes took place in the kidney tissue and showed similar to standard cyto-architecture.

* Tubular necrosis were observed although less compared to the test-1 group.

* NNSEE attenuated approximately 90\% of tubular necrosis in kidney.

* NNSEE showed better results than the test-1 group. 
Doxorubicin-induced kidney injury can lead to progressive renal fibrosis. The presented study, Hematoxyline and Eosin staining was used to assess tubular damage or tubular necorosis occurring in the kidney. The doxorubicin-induced model produced massive tubular damage in the positive control group. These fibrotic changes in doxorubicin-induced kidney was remarkably abrogated by NNSEE and the standard drug, indicating anti-fibrotic activity. Collectively, these data suggest that the NNSEE treatment improves the long-term outcome of injured kidney by ameliorating renal fibrosis.

\section{DISCUSSION}

In the presented study it was observed that the treatment by NNSEE exerted beneficial effects on renal fibrosis induced with Doxorubicin and Unilateral Ureter Obstruction (UUO) models. The results showed that the accumulation of fibrocytes, hyalinization of glomeruli in the kidney was evidently suppressed by NNSEE treatment. The possible underlying mechanism might be its inhibition of renal expression of TGF- $\beta_{1}$ and MCP-1 chemoattractants contributing to the fibrocytes recruitment into the injured kidney [8].

Renal fibrosis underlies all forms of end-stage renal disease. It is the inevitable consequence of an excessive accumulation of extracellular matrix that occurs in virtually every type of chronic kidney disease [9]. The incidence of chronic kidney disease leading to end-stage renal disease (ESRD) continues to increase worldwide. Almost all forms of ESRD are preceded by the progressive appearance of renal fibrosis. Currently, treatment of renal fibrosis is severely limited and often ineffective [10,11]. During the past decade, many drugs have been introduced onto the market for the management of renal fibrosis; their effectiveness, however, was unequivocal among the entire range of the population suffering from this renal disorder. Although most of these medications are safe and efficacious, in some patients prolonged usage may have questionable efficacy and cause disabling side-effects. This fact initiates the development of anti-fibrosis drugs derived from herbal sources and which have a better rate of success, ability to achieve full remission, with minimal incidence of side-effects. Alternative treatments using herbal medicines are therefore of great interest. The use of herbal medicines is now widespread for the treatment of various disease and disorders. The presented study focused on a most common and chronic disease - renal fibrosis, and its treatment using herbal extracts from a traditionally used herbal plant - Nelumbo nucifera.

A study was therefore designed to evaluate the anti-fibrosis activity in kidney by using NNSEE. Nelumbo nucifera is a herbaceous, aquatic plant of the Nelumbonaceae family, native to Tropical Asia and Queensland, Australia. It has been used in folk medicine and has been proved to have anti-obesity, anti-diabetic, anti-fertility, hepatoprotective, pulmonary protective, anti-parkinsonism, anti-bacterial, anti-oxidant, anti-platelet, anti-wrinkle, cytoprotective, analgesic, anti-proliferative and anti-inflammatory properties [12]. It is also used in the management of fibrosis but its pharmacological mechanism on renal fibrosis remains unknown. Through ongoing investigation in this area, the authors of the presented study found that the Nelumbo nucifera seed has anti-fibrotic activity in kidney, although this is not yet been proved scientifically.
Table 1. Screening of Phytochemical constituents

\begin{tabular}{lccc}
\hline $\begin{array}{c}\text { S. } \\
\text { No. }\end{array}$ & $\begin{array}{c}\text { Phytochemical } \\
\text { constituents }\end{array}$ & $\begin{array}{c}\text { Pet. ether extract of } \\
\text { Nelumbo nucifera seed }\end{array}$ & $\begin{array}{c}\text { Ethanolic extract of } \\
\text { Nelumbo nucifera seed }\end{array}$ \\
\hline 1. & Alkaloids & - & + \\
\hline 2. & Flavonoids & + & + \\
\hline 3. & Carbohydrates & + & + \\
\hline 4. & Proteins and amino acids & + & + \\
\hline 5. & Saponins & - & + \\
\hline 6. & Tannins & - & + \\
\hline 7. & Phenols & - & + \\
\hline 8. & Glycosides & - & + \\
\hline 9. & Polysterols & + & + \\
\hline
\end{tabular}

+Present; - Absent

Preliminary phytochemical investigations (Tab. 1) of NNSEE has showed positive results to alkaloids, saponins, tannins, flavonoids, carbohydrates, proteins and amino acids. The presence of these biochemical constituents may show better therapeutic action to renal fibrosis [13]. An acute toxicity study for NNSEE was conducted as per OECD Guidelines 423 by using Wistar albino rats. Each group of animals treated with the NNSEE solution by the oral route did not result in signs or symptoms of acute toxicity or mortality within 24 hours and a further period for 14 days. The obtained data on toxicity studies of NNSEE (Tab. 2) did not show any lethality $\left(\mathrm{LD}_{50}\right)$ in rats weighing up to $2,000 \mathrm{mg} / \mathrm{kg}$. According to the OECD guidelines, a safe dose range, i.e. $1 / 5(400 \mathrm{mg} / \mathrm{kg}$ b.wt) as test- 2 dose and $1 / 10$ (200mg/kg b.wt) as test- 1 dose, was used for subsequent studies [14]. Moreover, the selection of the animal model may depend on the progression of the disease, since some models develop renal fibrosis rapidly, e.g. ureteral obstruction model (1-2 weeks), while others take several weeks, e.g. doxorubicin, Anti-Thyl or protein overload induced nephropathy. Furthermore, reproducibility in the level of disease is an important criterion in choosing the animal model. In the current study it was noted that UUO and Doxorubicin-inducing methods are used mostly as models for inducing fibrotic activity. Other methods are very complicated and time-consuming processes for inducing disease. Therefore, in this study, the two abovementioned methods of inducing the disease were selected and investigated.

Table 2. Acute toxicity studies of NNSEE

\begin{tabular}{ccccccc}
\hline Groups & $\begin{array}{c}\text { Dose } \\
(\mathrm{mg} / \mathrm{kg})\end{array}$ & $\begin{array}{c}\text { No. of } \\
\text { animals }\end{array}$ & $\begin{array}{c}\text { Signs of } \\
\text { toxicity }\end{array}$ & $\begin{array}{c}\text { Onset } \\
\text { of } \\
\text { toxicity }\end{array}$ & $\begin{array}{c}\text { Mortality } \\
\text { rate }\end{array}$ & $\begin{array}{c}\text { Duration } \\
\text { of study }\end{array}$ \\
\hline I & $5 \mathrm{mg} / \mathrm{kg}$ & 3 & $\begin{array}{c}\text { No signs of } \\
\text { toxicity }\end{array}$ & Nil & Nil & 14 days \\
\hline II & $50 \mathrm{mg} / \mathrm{kg}$ & 3 & $\begin{array}{c}\text { No signs of } \\
\text { toxicity }\end{array}$ & Nil & Nil & 14 days \\
\hline III & $300 \mathrm{mg} / \mathrm{kg}$ & 3 & $\begin{array}{c}\text { No signs of } \\
\text { toxicity }\end{array}$ & Nil & Nil & 14 days \\
\hline IV & $2,000 \mathrm{mg} / \mathrm{kg}$ & 3 & $\begin{array}{c}\text { No signs of } \\
\text { toxicity }\end{array}$ & Nil & Nil & 14 days \\
\hline
\end{tabular}

Unilateral Ureter Obstruction (UUO). This model is characterized by tissue loss, atrophy of tubular epithelial cells and development of tubulointerstitial inflammation 
and fibrosis [15]. It resembles conditions seen in human obstructive nephropathy. In the UUO model, one ureter is ligated to block the flow of urine from kidneys to the urinary bladder. The accumulated urine in the kidneys activates renal tubular cells, which eventually leads to fibrosis in the obstructed kidney, whereas the contralateral kidney can, to some extent, regulate renal function.

In this study, renal fibrosis was well established within 3 days. Abnormalities of renal functions in nephrotoxic rats induced by UUO are increased creatinine, BUN levels, decrease in total protein, glomerular filtration rate levels, tubular cell necrosis and glomerular hyalinization, which lead to profibrotic responses [16]. The rats were then treated with standard drugs (Pirfenidone $200 \mathrm{mg} / \mathrm{kg}$ b.wt, p.o) and NNSEE at dose of 200 and $400 \mathrm{mg} / \mathrm{kg}$ b.wt p.o in rat (180$200 \mathrm{~g}$ ). After treatment, the biochemical parameters like serum creatinine(Graph 5), BUN level attenuates (Graph 6), total protein(Graph 7) and GFR levels were elevated (Graph 8 ) in UUO- induced renal fibrosis. This revealed that the NNSEE test-2 (400mg/kg b.wt) showed a greater effect than the test-1 (200mg/kg b.wt), which indicates anti-fibrotic activity and is a potently dose dependent.

Doxorubicin or Adriamycin-induced nephropathy. This model is characterized by the development of proteinuria, focal segmental glomerulosclerosis and tubulointerstitial fibrosis. It mimics the proteinuric condition in patients, and is considered an experimental analogue of human focal glomerulosclerosis. Doxorubicin is an anti-neoplastic agent and causes nephrotoxicity through many mechanisms, such as by inducing oxidative stress and by affecting water and urea transporters in the renal medulla.

In the presented study, renal fibrosis was well established within 1-2 months of a single intravenous injection of doxorubicin $(4 \mathrm{mg} / \mathrm{kg}$ b.wt). Doxorubicin plays a major role in deterioration of the glomerular structure, proteinuria, tubular and interstitial inflammation, culminating renal fibrosis in rats. Biochemical parameters were evaluated, e.g. serum creatinine (Graph 1), BUN levels were elevated (Graph 2), while total protein (Graph 3)[17] and GFR levels were decreased (Graph 4); tubular cell necrosis in doxorubicininduced renal fibrosis After two months, rats were treated with the standard drug (Pirfenidone $200 \mathrm{mg} / \mathrm{kg}$ b.wt, p.o) and NNSEE at a dose of 200 and $400 \mathrm{mg} / \mathrm{kg}$ b.wt p.o. NNSEE ameliorated all the profibrotic responses. After treatment, NNSEE and pirfenidone reversed all the manifestations of renal fibrosis. NNSEE test-2 (400mg/kg b.wt) showed a better therapeutic effect than the test-1 (200mg/kg b.wt), which indicates anti-fibrotic activity and is potently dose dependent.

Antioxidants naturally present in the body play a role in preventing the development of free radicals and inhibit the oxidation of other molecules. Oxidation is a chemical reaction that can produce free radicals, leading to chain reactions that may damage cells. Cells are protected against oxidative stress by an interacting network of antioxidant enzymes [18]. Antioxidant enzymes, e.g. SOD, CAT and peroxidases, are the major enzymes which prevent free radicals. SOD is present in almost all aerobic cells and in extracellular fluids. In humans, SOD is present in cytosol and mitochondrion, and also exists in extracellular fluids. SOD catalyzes the breakdown of the superoxide anion (free radical) into oxygen and hydrogen peroxide [19]. CAT is mainly present in peroxisomes which catalyze the conversion
Table 3. Evaluation of Serum creatinine, BUN, Total protein and GFR levels in doxorubicin induced group

\begin{tabular}{|c|c|c|c|c|c|}
\hline $\begin{array}{l}\text { S. } \\
\text { No. }\end{array}$ & Goups & $\begin{array}{l}\text { Serum } \\
\text { creatinine } \\
(\mathrm{mg} / \mathrm{dL})\end{array}$ & $\begin{array}{c}\text { BUN level } \\
(\mathrm{mg} / \mathrm{dL})\end{array}$ & $\begin{array}{c}\text { Total } \\
\text { protein } \\
\text { level }(\mathrm{g} / \mathrm{dL})\end{array}$ & $\begin{array}{c}\text { GFR } \\
(\mathrm{mL} / \\
\left.\mathrm{min} / 1.73 \mathrm{~m}^{2}\right)\end{array}$ \\
\hline 1. & $\begin{array}{c}\text { Normal } \\
\text { Saline }(4 \mathrm{ml} / \mathrm{kg})\end{array}$ & $0.966 \pm 0.06$ & $17.66 \pm 0.66$ & $7.21 \pm 0.24$ & $106.7 \pm 2.431$ \\
\hline 2. & $\begin{array}{c}\text { Positive control } \\
\text { Doxorubicin } \\
(4 \mathrm{mg} / \mathrm{kg})\end{array}$ & $6.05 \pm 0.11^{\#}$ & $37.83 \pm 2.72^{\#}$ & $3.51 \pm 0.43^{\#}$ & $35.3 \pm 4.801^{\#}$ \\
\hline 3. & $\begin{array}{c}\text { Standard } \\
\text { Pirfenidone } \\
(200 \mathrm{mg} / \mathrm{kg})\end{array}$ & $2.117 \pm 0.01^{\mathrm{b}}$ & $29.66 \pm 1.90^{b}$ & $5.83 \pm 0.47^{b}$ & $93.3 \pm 3.145^{c}$ \\
\hline 4. & $\begin{array}{c}\text { Test-1 } \\
\text { NNSEE } \\
(200 \mathrm{mg} / \mathrm{kg})\end{array}$ & $3.517 \pm 1.00^{\mathrm{a}}$ & $30.63 \pm 0.66^{b}$ & $4.97 \pm 0.31^{\mathrm{a}}$ & $53.3 \pm 3.894^{a}$ \\
\hline 5. & $\begin{array}{c}\text { Test-2 } \\
\text { NNSEE } \\
(400 \mathrm{mg} / \mathrm{kg})\end{array}$ & $2.833 \pm 1.12^{\mathrm{b}}$ & $30.33 \pm 0.66^{b}$ & $5.02 \pm 0.43^{\mathrm{a}}$ & $75.8 \pm 3.567^{c}$ \\
\hline
\end{tabular}

All values are expressed as mean $\pm \operatorname{SEM},(n=6)$. Statistical significance ${ }^{a} p<0.05,{ }^{b} p<0.01$, ' $p<0.001$, was compared with positive control group (One-way ANOVA followed by Dunnett's multiple comparisons test).

Table 4. Evaluation of Serum creatinine, BUN, Total protein and GFR levels in UUO induced group

\begin{tabular}{cccccc}
\hline $\begin{array}{c}\text { S. } \\
\text { No. }\end{array}$ & Groups & $\begin{array}{c}\text { Serum } \\
\text { creatinine } \\
(\mathrm{mg} / \mathrm{dL})\end{array}$ & $\begin{array}{c}\text { BUN level } \\
(\mathrm{mg} / \mathrm{dL})\end{array}$ & $\begin{array}{c}\text { Total } \\
\text { protein } \\
\text { level }(\mathrm{g} / \mathrm{dL})\end{array}$ & $\begin{array}{c}\mathrm{GFR}(\mathrm{mL} / \\
\left.\mathrm{min} / 1.73 \mathrm{~m}^{2}\right)\end{array}$ \\
\hline 1. & Normal $(4 \mathrm{ml} / \mathrm{kg})$ & $0.96 \pm 0.06$ & $17.57 \pm 0.76$ & $7.48 \pm 0.24$ & $103.00 \pm 2.944$ \\
\hline & $\begin{array}{c}\text { Positive control } \\
\text { (Doxorubicin } \\
4 \mathrm{mg} / \mathrm{kg})\end{array}$ & $7.68 \pm 0.92^{\#}$ & $40.16 \pm 2.93^{\#}$ & $2.08 \pm 0.63^{\#}$ & $40.30 \pm 3.801^{\#}$ \\
2. & $\begin{array}{c}\text { Standard } \\
\text { (Pirfenidone }\end{array}$ & $4.46 \pm 0.42^{\mathrm{b}}$ & $30.16 \pm 1.07^{\mathrm{b}}$ & $6.21 \pm 0.93^{\mathrm{b}}$ & $90.35 \pm 3.145^{\mathrm{c}}$ \\
\hline 3. & & & & \\
\hline 4. & $\begin{array}{c}\text { Tent-1 } \\
\text { (NNSEE 200mg/kg) }\end{array}$ & $5.26 \pm 0.59^{\mathrm{a}}$ & $31.54 \pm 1.76^{\mathrm{a}}$ & $4.90 \pm 0.95^{\mathrm{a}}$ & $60.70 \pm 5.357^{\mathrm{b}}$ \\
\hline 5. & $\begin{array}{c}\text { Test-2 } \\
\text { (NNSEE 400 mg/kg) }\end{array}$ & $4.85 \pm 0.40^{\mathrm{b}}$ & $30.66 \pm 0.80^{\mathrm{b}}$ & $5.91 \pm 0.67^{\mathrm{b}}$ & $81.58 \pm 3.498^{\mathrm{c}}$ \\
\hline
\end{tabular}

All values are expressed as mean $\pm \operatorname{SEM},(n=6)$. Statistical significance a $p<0.05,{ }^{b} p<0.01$, ' $p<0.001$, was compared with positive control group (One-way ANOVA followed by Dunnett's multiple comparisons test).

Table 5. Evaluation of Invivo antioxidant levels (SOD and CAT)

\begin{tabular}{lccc}
\hline $\begin{array}{l}\text { S. } \\
\text { No. }\end{array}$ & Groups & $\begin{array}{c}\text { SOD } \\
\text { (Units } / \mathrm{mg} \\
\text { protein) }\end{array}$ & $\begin{array}{c}\text { CAT } \\
\text { (Units/mg } \\
\text { protein) }\end{array}$ \\
\hline 1. & Normal (Saline $4 \mathrm{ml} / \mathrm{kg})$ & $305.10 \pm 4.64$ & $78.13 \pm 1.81$ \\
\hline 2. & Positive control & $180.56 \pm 8.25^{\#}$ & $25.83 \pm 2.54^{\#}$ \\
\hline 3. & Standard (Pirfenidone $200 \mathrm{mg} / \mathrm{kg})$ & $212.32 \pm 4.96^{\mathrm{b}}$ & $40.65 \pm 3.26^{\mathbf{b}}$ \\
\hline 4. & Test-1 (NNSEE $200 \mathrm{mg} / \mathrm{kg})$ & $206.65 \pm 3.96^{\mathrm{b}}$ & $38.56 \pm 2.67^{\mathrm{b}}$ \\
\hline 5. & Test-2 (NNSEE $400 \mathrm{mg} / \mathrm{kg})$ & $209.84 \pm 3.65^{\mathrm{b}}$ & $39.52 \pm 2.35^{\mathrm{b}}$ \\
\hline
\end{tabular}

All values are expressed as mean $\pm \operatorname{SEM},(n=6)$. Statistical significance a $p<0.05,{ }^{b} p<0.01$, ${ }^{c} p<0.001$, was compared with positive control group (One-way ANOVA followed by Dunnett's multiple comparisons test)

of hydrogen peroxide to oxygen and water by using iron or manganese cofactors [20]. Peroxidases also catalyze the reduction of hydrogen peroxides [21].

In the current study, the antioxidant activity of Superoxide dismutase (SOD) and Catalase (CAT) was estimated. Oxidative stress is produced by doxorubicin which induces glomerular toxicity in rats. Redox cycling of the quinone 
functional group doxorubicin is proposed as the key factor in doxorubicin nephrotoxicity. Reactive oxygen species (ROS) may initiate a degenerative cascade by the oxidation of cellular thiols and lipid membrane structures. Doxorubicin has been suggested to upregulate NADPH-oxidase (NOX), an important source of ROS in the kidney [22]. The generated reactive oxygen species, such as superoxide and hydroxyl radicals, have the potential to cause damage to various intracellular components. A deficiency of oxygen supply may destroy the tubular cells and glomerular cells in kidney that leads to one of the reason for renal fibrosis. SOD activity (Graph 9) decreases in renal fibrosis-induced animals due to excessive formation of superoxide free radicals, and also the activities of scavenging enzyme CAT (Graph10) significantly.

UUO-induced and doxorubicin-induced animals showed a significant reduction of Superoxide dismutase (SOD) and Catalase (CAT) enzymes, compared to the normal group of rats. Elevated reactive oxygen species may prompt the disease. The induced animals were treated with NNSEE at a dose of 200 and $400 \mathrm{mg} / \mathrm{kg}$ b.wt which reversed all these manifestations after the experimental period. This may be due to the presence of polyphenols and alkaloids in the NNSEE which have an antioxidant property and nephroprotective potential in a dose dependent manner. The test $-2(400 \mathrm{mg} / \mathrm{kg})$ of NNSEE showed a more potent therapeutic activity than the test-1 (200mg/kg).

In the histopathology of kidney tissue, it was clearly noted that hyalinization of glomeruli, degenerative changes, tubular damage, vacuolization and necrosis took place, which all led to renal fibrosis [23]. Rats were then treated with the NNSEE, as well as the standard drug Pirfenidone, which ameliorated all the profibrotic responses, the regeneration of glomerular cells took place, and excess hyalinalized glomeruli decreased. NNSEE of test-2 (400mg/kg b.wt) showed better therapeutic activity than the test-1 (200mg/kg b.wt) in a dose dependent manner, which may be due mainly to the presence of alkaloids and polyphenols.

Hence, the presented study shows that NNSEE has an anti-fibrotic property in kidney by altering the profibrotic responses, and also showed antioxidant property by increasing SOD and CAT levels, which may be due to the presence of constituents (alkaloids and polyphenols) present in the Nelumbo nucifera seed. These results suggest that NNSEE could be effective in reversing the profibrotic responses in renal fibrosis. The test-2 $(400 \mathrm{mg} / \mathrm{kg})$ of NNSEE showed greater therapeutic effect than the test-1 $(200 \mathrm{mg} / \mathrm{kg})$.

\section{CONCLUSION}

This research on renal fibrosis using doxorubicin and UUOinducing models strongly implies that the over-production of hyalinized glomeruli may be due to the activation of TGF- $\beta$, which causes repeated tissue injury leading to renal fibrosis. NNSEE ameliorates renal fibrosis by inhibiting the recruitment of fibrocytes into the kidney, and influences antioxidant and anti-fibrotic activity. This substantiates the therapeutic utility in renal fibrosis. The results obtained suggest that NNSEE might be a potential agent to be applied in clinics for preventing the progression of renal fibrosis in a dose dependent manner. Further studies are needed to investigate the role of NNSEE in other models of renal fibrosis, such as subtotal nephrectomy, renal ischemia, and
Anti-Thyl antibody for the exact biochemical constituents responsible for anti-fibrotic activity.

\section{Acknowledgement}

The authors express their gratitude to the management of Krishna Teja Pharmacy College, Chadalawada Nagar, Tirupati, Chittoor district, Andhra Pradesh, India, for supported in the performance of this study.

\section{REFERENCES}

1. Hideki Yokoi, Suqawara, Mukoyama, Mori, Makino, Suqanami, Naqae, Yahata, Fujinaga, Tanaka, Nakao.Role of connective tissue growth factor in profibrotic action of transforming growth factor- $\beta$ : A potential target for preventing renal fibrosis. American Journal of Kidney Disease 2011; 38(4) (suppl 1): 134-138.

2.Patricia silva 2015, Death report of kidney failure linked to fibrosis. Available from http://ckdnews.com/2015/08/10/study-reports-newpotential-therapy-kidney-fibrosis-ckd/

3. ArvindAgarwal, Deepika Bora, ChanderpriyaAgarwal, Ratendra Kumar and VeermaramChoudhary. CNS stimulant and antidepressant activity of seeds of Abelmoschusesculentus in rats. Bull. Pharm. Res. 2015; 5(2): 1-4.

4. SirishaChowdary. Neuropharmacological Screening of the ethanolic extract of Nelumbo nucifera seeds. International Journal in Pharmacy and Biotechnology 2013; 1(5): 635-642.

5. Gupta. Drug screening methods (preclinical evaluation of new drugs). International Students Edition ( $2^{\text {nd }}$ edition) 2009; chapter 11: 185-193.

6. Claiborne and Malinowski and Fridovich. Purification and characterization of hydroperoxidase II of Escherichia coli B. The Journal of Biological Chemistry 1979; 254(22): 11664-11668.

7. Mishra and Fridovich. The role of superoxide anion in the autooxidation of epinephrine and a simple assay for superoxide dismutase; The Journal of Biological Chemistry; 247: 3170-3175.

8. Oka, Suwitra and Soebadi. Obstructive nephropathy of kidney stone: The role of caspase-3, TGF- $\beta$ and TNF- $\alpha$ in kidney fibrosis. Bali Medical Journal 2014; 3(2): 104-111.

9. Campanholle, Liqresti, Gharib and Duffield. Cellular mechanism of tissue fibrosis: Novel mechanism of kidney fibrosis. American Journal of Physiology 2013; 304: 591-603.

10. Decleves\& Sharma. Novel targets of anti-fibrotic and anti-inflammatory treatment in CKD. Nature Reviews Nephrology 2014; 10: 257-267.

11.Leung, Tonelli and James. Chronic kidney disease following acute kidney injuryrisk and outcomes. Nat. Rev. Nephrol 2013; 9: 77-85.

12. Bharadwaj and Modi. A review on therapeutic potential of Nelumbo nucifera: the sacred lotus. International Journal of Pharmaceutical Sciences and Research 2016; 7(1): 42-54.

13. PrashantTiwari, Bimlesh Kumar, MandeepKaur and Harleen Kaur. Phytochemical screening and extraction: A Review. Internationale Pharmaceutica Sciencia 2011; 1(1): 98-106.

14. Vidya, Ramesh, Rajashekar, Meghana, Nazeer. The nephroprotective activity of methanolic extracts of Phyllanthusacidus leaves against gentamycin induced nephrotoxicity in experimental rodents. International Journal of Pharmacy and Pharmaceutical Sciences 2013; 5(4): 209-213.

15. Klahr and Morrissey. Obstructive nephropathy and renal fibrosis. Am J Physiol Renal Physiol 2002; 283: 861-875.

16. Luis Pimentel, Angel Montero, Susheng Wang, Igor Yosipiv, Samir Dhar and Manuel Martinez Maldonado. Sequential changes in renal expression of renin-angiotensin system genes in acute unilateral obstruction. Kidney International 1995; 48(4): 1247-1253.

17. Manimala, Karpagam, Deecaraman, Clement Atlee, PurushothPrabu. Evaluation of nephroprotective and antioxidant activity of ethanolic extracts of Momordicadioica leaves. Der Pharmacia Lettre Journal 2015; 7(4): 153-156.

18. Davies KJ. Oxidative stress: The paradox of aerobic life. Biochemical Society Symposium 1995; 61: 1-31.

19. Bannister, Rotilio, Bannister. Aspects of the structure, function and applications of superoxide dismutase. CRC Critical reviews in Biochemistry 1987; 22(2): 111-80.

20. Chelikani, Fita and Loewen. Diversity of structures and properties among catalases. Cellular and Molecular Life Sciences 2004; 61(2): 192-208. 
21. Rhee, Ho Zoon Chae and Kanghwa Kim. Peroxiredoxins: a historical overview and speculative preview of novel mechanisms and emerging concepts in cell signaling. Free radical biology and Medicine 2005; 38(12): 1543-1552.

22. CsabaImreSzalay, KatalinErdelyi, Gabor Kokeny, EnikoLajtar, Maria Godo, CsabaRevesz, TamasKaucsar, Norbert Kiss, Marta Sarkozy, Tamas Csont, Tibor Krenacs, Gabor Szenasi, Pal Pacher and Peter Hamar.
Oxidative or Nitrative stress and inflammation drive progression of doxorubicin induced renal fibrosis in rats as revealed by comparing a normal and fibrosis resistant rat strain. Journal Pone 2015; 10(6): 1-17. 23. Alton Farris and Robert Colvin. Renal interstitial fibrosis: Mechanism and evaluation. Current Opinion in Nephrology and Hypertension 2012; 21(3): 289-300. 